\title{
Step energies and equilibrium shape of strained monolayer islands
}

\author{
J.E. Prieto ${ }^{1}$ and I. MARKov ${ }^{2}$
}

1 Centro de Microanálisis de Materiales, Departamento de Física de la Materia Condensada, Condensed Matter - Physics Center (IFIMAC) and Instituto "Nicolás Cabrera", Universidad Autónoma de Madrid, E-28049 Madrid, Spain

Institute of Physical Chemistry, Bulgarian Academy of Sciences, 1113 Sofia, Bulgaria

PACS 68.35.Md - Surface thermodynamics, surface energies

PACS 81.10.Aj - Theory and models of crystal growth; physics and chemistry of crystal growth, crystal morphology, and orientation

PACS 68.43.Hn - Structure of assemblies of adsorbates (two- and three-dimensional clustering)

\begin{abstract}
Using a simple atomistic model of anharmonic nearest-neighbors interaction, we have calculated the step energies of strained hexagonal monolayer islands. These have been found to decrease with the absolute value of the misfit due to the strain relaxation at steps. The effect is significantly more pronounced in the case of positive misfit owing to the stronger repulsive interatomic forces. Furthermore, (111)-faceted steps are favored at positive misfit (compressed islands) and, to a lesser extent, (100)-faceted steps at negative misfits (tensile islands). The result is rationalized in terms of the different bonding geometries at step edges and a comparison with experiments is included. Thus, the equilibrium shape transforms from regular hexagons at zero misfit to threefold symmetric hexagons with increasing misfit.
\end{abstract}

The problem of the equilibrium shape of crystals has a long and venerable history [1]5] (for a review see Ref. [6]). In recent decades this problem in connection with epitaxial stress has attracted much attention in the case of the formation of self-assembled quantum dots [7-10]. The equilibrium shape of two-dimensional monolayer-high islands has also emerged as an important topic in connection with facet-shape changes of three-dimensional crystallites [6], the possibility of measuring the absolute values of the step and kink formation energies [1] and the growth of crystal surfaces 12 .

The initial stages of the epitaxial growth of thin films include the formation and growth of elastically-strained monolayer islands [13, 14]. This has motivated an interest in the study of their equilibrium shapes 15 18. These authors have addressed the behavior of the total energy of rectangular islands, considered to be given by the sum of strain and step energies, the latter being assumed as strain-independent [15]17. The systems studied are characterized by a highly anisotropic surface stress, as for example, reconstructed, single-domain $\mathrm{Si}(100)$ terraces. But also for the case of isotropic epitaxial strain, authors find an island shape instability via long-range step-step interaction even in the absence of step-energy anisotropy [15]17].
On the contrary, in the present Letter we consider islands on highly symmetric sixfold coordinated surfaces, for which the surface stress tensor is isotropic [19] and we set out to study the contribution of strain, not considered before, to the step energies and in turn to the aspect ratio. We chose to use the simplest model that contains the essential ingredients to describe the effect of strain. We perform atomistic calculations using model potentials for simulating hexagonal islands at $T=0 \mathrm{~K}$. Our approach follows the spirit of the work of Hamilton on overlayer strain relief in $f c c(100)$ surfaces $[20$. As known, hexagonal islands are enclosed by steps of two different types: (100)-faceted or $A$ steps and (111)-faceted or $B$ steps which, when circulating along the island perimeter, follow the alternating sequence $B A B A B A$ (see Fig. 10). In these highly symmetric surfaces, equilibrium island shapes are expected to be largely determined by the ratio $r=\gamma_{111} / \gamma_{100}$ of the free energies of $B$ and $A$ steps. For some homoepitaxial systems, this ratio has been studied in detail (results are summarized in Ref.[ [11]]). In general $r$ differs from unity. A relatively large deviation $\left(r^{-1}=1.15\right)$ has been reported for vacancy islands on $\operatorname{Pt}(111)$ by Michely and Comsa 21 from the measured ratio of the step lengths.

We consider an atomistic model of a rigid substrate with 


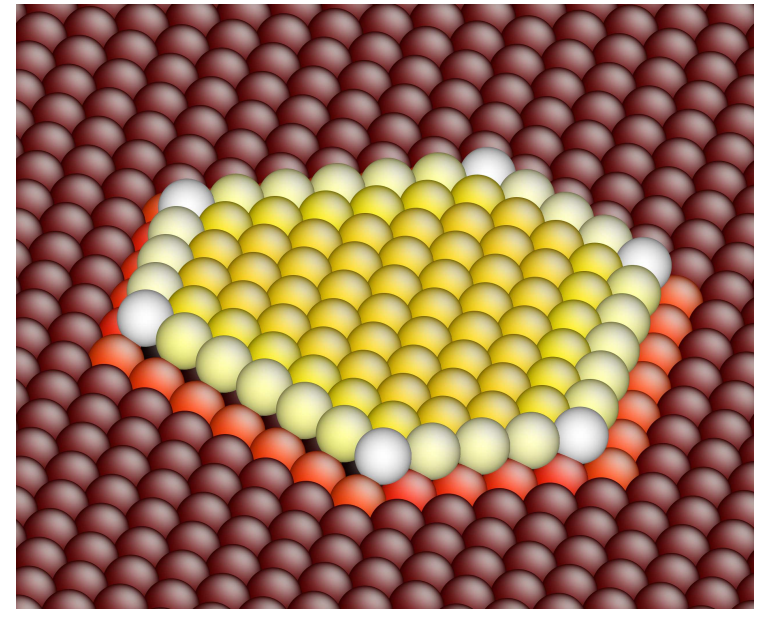

Fig. 1: Hexagonal, threefold symmetric island of 5 atoms in the (111)-faceted, $A$ steps and 7 atoms in the (100)-faceted, $B$ steps $(5,7)$. The color scale denotes the height of the considered atom and has been represented using the AtomEye software 22. This height is measured above the level of the corresponding crystallographic plane, but a constant fraction of the interlayer distance has been added in order to better distinguish atoms from different levels. The height is biggest at edges and corners due to the atoms "climbing up" on their neighbours underneath due to strain relaxation. The lattice misfit is $-7 \%$.

sixfold symmetry and lattice parameter $a$ that can represent $f c c(111)$ or $h c p(0001)$ surfaces on which monolayer islands grow. Atoms interact in the first coordination sphere through an anharmonic Morse potential 23 .

$$
V(r)=V_{0}\left[e^{-12(r-b)}-2 e^{-6(r-b)}\right],
$$

where $b$ is the equilibrium atom separation, so the lattice misfit is given by $\varepsilon=(b-a) / a$. For every configuration, the cluster is allowed to relax by varying iteratively the atomic positions until all the forces fall below some negligible cutoff value. Clearly, our atomistic model is intentionally oversimplified in order to study the effect of the lattice misfit in its pure form. It obviously gives a regular hexagon, $l_{100}=l_{111}$, as the equilibrium island shape at zero misfit.

We calculate the values of the energies of $A$ and $B$ steps by means of the following procedure. The total energy of a strained polygonal island contains contributions from the interior bonds, which are the most highly strained due to the lattice misfit, and from the edges and corners, where strain is relaxed. In order to compute the step energy of a regular-hexagonal island of $(n, n)$ atoms at the edges (where the first and second numbers denote the number of atoms in the $B$ and $A$ edges, respectively) we consider the two configurations $(n+1, n)$ and $(n-1, n)$. We then subtract the energies of the two islands and correct for the different number of atoms contained by adding the energy of the corresponding number of completely strained "interior" atoms at the center, a contribution that can be calculated analytically from the known interaction poten- tial, [Eq. (10]. The corner energies can be assumed to be the same in both islands if they are large enough, so that the energy difference gives the increase in edge energy due to the increase of the length of the $B$ steps. The edge energy of $A$ steps can be calculated analogously by comparing $(n, n+1)$ and $(n, n-1)$ islands. It is clear that in this way we are considering all effects related to strain relaxation at edges to be included into the step energy. This makes sense since otherwise the only contribution to the step energy would be the number of broken bonds which, being the same for $A$ and $B$ steps, would result in no difference in their edge energies. The results of these calculations are shown in Fig. 2 ,

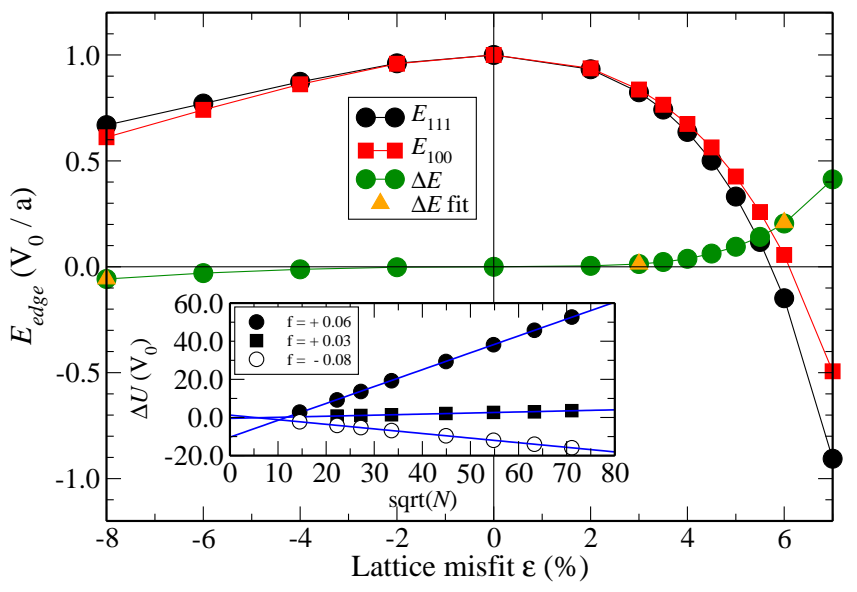

Fig. 2: Dependence of the energies of $A$ and $B$ steps, in units of $V_{0} / a$, on the lattice misfit. The third curve represents the difference of the step energies. The triangular points are the values calculated from triangular islands, as shown in the insert. Insert: Difference of the total energies of triangular islands, in units of $V_{0}$, as a function of the square root of the total number of atoms. These islands are enclosed by only either $A$ or $B$ steps depending on the orientation. Results for three values of the lattice misfit are given: 3,6 and $-8 \%$.

Since this is a point of crucial importance, we now proceed to check our assumption that strain affects the edge energies. In order to eliminate the effect of the "interior" strain, we compare the total energies of triangular islands of the same size, but enclosed by either only $A$ or only $B$ edges. Because of their equal sizes, both types must contain equal contributions of "interior" strain and can only differ in the way strain is relaxed at edges of different types. The energy difference of such islands is then given by

$$
\Delta U=3 \Delta E_{\text {corner }}+3\left[(\sqrt{2 N}-1 / 2)-2 l_{c}\right] \Delta E_{\text {edge }}
$$

where $\sqrt{2 N}-1 / 2$ is the full length of the edge expressed as a function of the total number $N$ of atoms in the island (here a factor $1 / 4$ has been neglected compared to $2 N$ ), the term in square brackets gives an "effective" length of the edge by subtracting an extension $l_{c}$ around each corner where edge energies are affected and finally, $\Delta E_{\text {edge }}=$ 
$\gamma_{100}-\gamma_{111}$ is the difference in energies between the two types of steps.

The insert of Fig. 2 shows the difference of the energies of triangular islands enclosed by either $A$ or $B$ steps at three different values of the misfit, $3 \%$ and $6 \%$ (compressed islands), and $-8 \%$ (tensile islands). The data can be very well described by straight lines, confirming the validity of the considerations above. From the slopes, the difference in edge energies $\Delta E_{\text {edge }}$ can be calculated. The slope is positive for compressed islands $\left(\gamma_{100}>\gamma_{111}\right)$ and negative for islands under tensile stress $\left(\gamma_{100}<\gamma_{111}\right)$, in agreement with Fig. 2. Furthermore, the result is in very good quantitative agreement with the value obtained by subtracting the values of $\gamma_{100}$ and $\gamma_{111}$ calculated directly, as shown in Fig. 2, giving consistency to our procedure. In addition, to the extent that the difference $\Delta E_{\text {corner }}$ in corner energies between the two types of triangular islands can be neglected, the extension $l_{c}$ of the region at the step affected by the corner can be calculated from the $y$-intercept of the lines in the insert of Fig. 2. We obtain the values of 5.6 and 8.0 atomic spacings for $3 \%$ and $6 \%$ misfit, respectively, and 3.4 spacings for $-8 \%$. These results appear very reasonable, of the order of a few lattice spacings, increasing with misfit and larger for positive than for negative values of the misfit, because of the more efficient relaxation at edges and corners 24 .

Figure 2 demonstrates our main result, the behavior of the step energies, $\gamma_{100}$ and $\gamma_{111}$, as a function of misfit. A number of relevant facts can be observed. The energy of both types of steps has a value of $V_{0} / a$ at zero misfit in accordance with dangling-bond counting. We see that both edge energies decrease with increasing absolute value of the lattice misfit, as expected due to strain relaxation at island edges, but the effect is much stronger in the case of compressive misfits because of the more effective relaxation: The region with relaxed bonds extends significantly longer into the island in the case of positive misfits [25]. Another point, which will be addressed below, is that the energies become negative for high enough positive values of the misfit.

A very important feature visible in Fig. 2 is the opposite sign of the difference between the step energies of $A$ and $B$ steps. For compressed islands, i.e. for positive values of the misfit, $B$-type, (111)-faceted steps are significantly favored, while $A$-type, (100)-faceted ones have lower energy for negative values of the misfit, although the effect is smaller. So (111)-faceted steps will be favored for positive and (100)-faceted steps for negative misfits. This is a clear prediction of our model and can be rationalized as follows: A compressed island relaxes strain at steps by the displacement of edge atoms (from the bottoms of the corresponding potential troughs they would occupy in the absence of misfit) in the outward direction [26]. For (111)-faceted steps, this means that edge atoms are pushed towards "bridge" positions of the substrate layer underneath, while for (100)-faceted steps, they are pushed towards "on-top" positions (see Fig 1). Sensibly, the former situation has a lesser cost in bonding (adhesion) energy than the latter. The situation is clearly reversed in the case of negative misfits, i.e. in tensile islands, for which strain relaxation at steps forces edge atoms to be displaced in the inward direction. Now in this case, atoms at (111)-faceted steps are pushed towards "on-top" positions and atoms at (100)faceted steps towards less unfavorable "bridge" positions.

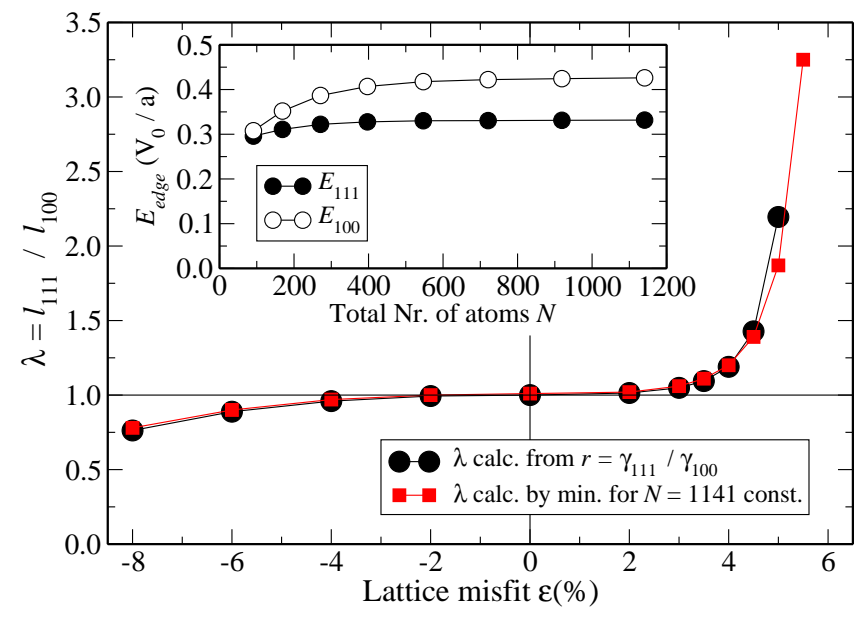

Fig. 3: Dependence of the ratio of the step lengths (the equilibrium shape) of $A$ and $B$ steps on the lattice misfit, calculated both from the differences in edge energies obtained from Fig. 2 and from a direct search of the minimum energy among islands of nearly constant number of atoms (see text). Insert: Dependence of the energies of $A$ and $B$ steps on island size.

Figure 3 gives the aspect ratio of the step lengths $\lambda=$ $l_{111} / l_{100}$, characterizing the equilibrium shape of the islands, as a function of the misfit. Data in this plot have been determined in two different ways: First, from the calculated values of the step energies $\gamma_{111}$ and $\gamma_{100}$ (shown in Fig. 2), the aspect ratio $\lambda$ has been obtained by minimization of the perimeter energy at constant number of atoms (island area), as given by

$$
\lambda=\frac{r-2}{1-2 r}
$$

where $r=\gamma_{111} / \gamma_{100}$ is the ratio of the step energies, obtained from Fig. 2. The second procedure is the direct calculation of the total energy of islands of different lengths $l_{100}$ and $l_{111}$ but with constant total number of atoms to find the configuration of minimum energy. Since this is not possible to achieve exactly for a large enough number of different configurations with integer number of atoms, several ones containing numbers very close to the desired size (e.g. 1141 atoms) were selected and the minimum-energy configuration was determined by interpolation. Figure 3 shows that the two procedures give basically the same results, confirming the validity of the approach. We see that the (111)-faceted step becomes several times longer that the (100)-faceted one at positive misfit. The opposite tendency is observed for tensile islands, although the effect is smaller. 
The question of the negative edge energies at high positive values of the misfit can now be addressed. Considering Eq. (3), it is immediately seen that for $r \rightarrow 1 / 2$, $\lambda$ tends to infinity, meaning that the threefold symmetric hexagon has transformed into a triangle exposing only $B$ steps. An inspection of Fig. 2 shows that negative edge energies appear for misfits higher than about $5.5 \%$ in our simplified model. For those values of the misfit, Fig. 3 predicts already a factor $\lambda$ of the order of 3 . This means that negative edge energies, which would imply an instability towards disintegration of large islands into many small islands, are reached at very high values of the misfit, together with a clear preference for a given type $(B)$ of steps. For those values, real systems will probably relax strain by some mechanism not considered in our model, such as the introduction of misfit dislocations.

The insert of Fig. 3 shows the dependence of the edge energies on island's size. As seen the energy is sizeindependent with the exception of very small sizes where the elastic fields at the edges substantially overlap and the contribution of corners becomes significant. This is in accordance with the interpretation of the edge energy as a macroscopic concept. Finally, Fig. 4 shows the polar plot $\gamma(\theta)$ together with the Wulff construction for the determination of the equilibrium shape of an island compressed at $5 \%$. For this plot, in addition to the edge energies $\gamma_{111}$ and $\gamma_{100}$ (and the corresponding $\gamma_{\overline{1} 13}$, which does not appear in the equilibrium shape) the kink energies $\beta$ in those steps were calculated in order to obtain through

$$
\gamma(|\theta|)=\gamma(0) \cos (|\theta|)+\frac{\beta}{a} \sin (|\theta|),
$$

in complete analogy with the three-dimensional polar plot 27, the dependence of the step energy on orientations close to that of a smooth, kinkless step (of energy $\gamma(0))$ with its corresponding singular point in the $\gamma(\theta)$ polar plot.

The most important conclusion of our work is that compressed islands are predicted to possess an equilibrium shape containing longer (111)-faceted steps. On the contrary, tensile islands will show a nearly regular hexagon as the equilibrium shape, with a slight preference for (100)faceted steps, as the step energy difference is very small. Concerning the limitations of our approach, it is important to be aware that we are considering only the effect of strain in the equilibrium shape of epitaxial monolayer islands on hexagonal substrates. Therefore, our model is best suited for the description of simple metallic systems. However, other aspects neglected in our simulations are found to play only minor roles determining equilibrium shapes in our conditions. For example, the assumption of a rigid substrate was checked in simulations allowing a number of layers of the substrate to relax. Figure 5 shows the energies of $A$ - and $B$-type steps calculated by allowing one monolayer of the substrate to relax in the same way as the atoms in the island, together with the results for a rigid substrate shown in Fig. 2. The result is that the dif-

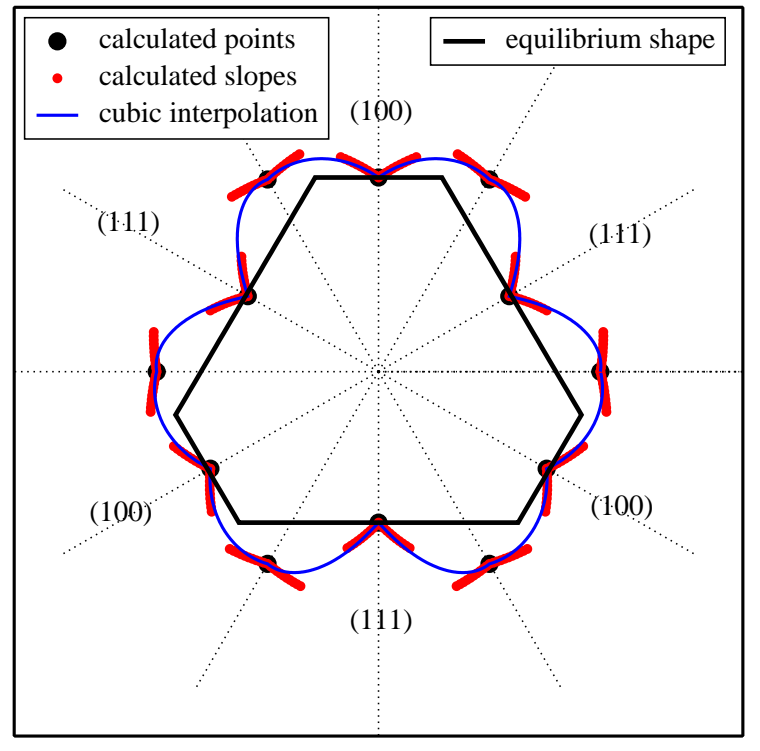

Fig. 4: Polar plot $\gamma(\theta)$ and Wulff's construction of an island under compression of $5 \%$.

ference in energies of the two type of edges is very similar in both cases. We conclude that the contribution of elastic interactions mediated by the substrate does not significantly influence equilibrium shapes for the relatively large island sizes and the highly (threefold) symmetric substrate considered here. Also the assumption of different bonding strengths between island atoms (cohesion) and between island and substrate atoms (adhesion) does not significantly affect island shapes, as was checked by simulations for different values of the adhesion parameter between island and substrate. This confirms that our simulations contain the essential ingredient determining equilibrium shapes, the different lattice parameters of film and substrate materials. Clearly, equilibrium shapes can be affected by several additional factors not considered here, such as the presence of dislocation networks, surface reconstructions, stacking faults, interdiffusion with the substrate, changes in stoichiometry of compounds, etc. These factors also explain the scarcity of reliable examples of experimental results to compare with. Some of them are discussed in the following.

Clear equally oriented threefold hexagonal islands with longer (111)-faceted steps have been reported as the equilibrium shape in the epitaxial system $\mathrm{Ag} / \mathrm{Pt}(111)$ with a compressive stress of $4.3 \%$ [28]. Authors calculate a ratio of step free energies $\gamma_{100} / \gamma_{111}=1.25$, which gives a ratio of edge lengths of about 2 , in agreement with our model (see Fig. 3). Another interesting system is $\mathrm{Co} / \mathrm{Pt}(111)$. Here, if deposition is performed at low temperatures to avoid interdifussion, quasi-hexagonal, monolayer islands are obtained as equilibrium shapes upon annealing [2930]. These islands show a network of partial dislocations on top as a result of an increase of the density of Co atoms, which turns the stress from tensile to compressive in the 


\begin{tabular}{|c|c|c|c|}
\hline System [Ref.] & Strain & $\begin{array}{c}\text { Shape } \\
\text { Pref. facet }\end{array}$ & Comm. \\
\hline \hline $\mathrm{Ag} / \mathrm{Pt}(111)[28]$ & $4.3 \%$ & $\begin{array}{c}\text { 3-f hex. } \\
(111)\end{array}$ & \\
\hline $\mathrm{Co} / \mathrm{Pt}(111)[30]$ & $\begin{array}{c}>0, \\
\text { recons. }\end{array}$ & $\begin{array}{c}\text { compact } \\
(111)\end{array}$ & recons. \\
\hline $\mathrm{Co} / \mathrm{Ru}(0001)[33]$ & $-7.3 \%$ & $\begin{array}{c}\text { triang. } \\
(100)\end{array}$ & $\begin{array}{c}\text { poss. } \\
\text { kinet. }\end{array}$ \\
\hline $\mathrm{Cu} / \mathrm{Cu}(111)[11]$ & $\sim 0$ & $\sim 6$-f hex. & \\
\hline $\mathrm{Ag} / \mathrm{Ag}(111)[11]$ & $\sim 0$ & $\sim 6$-f hex. & \\
\hline $\mathrm{Au} / \mathrm{Au}(111)[21]$ & $\begin{array}{c}>0, \\
\text { recons. }\end{array}$ & $\begin{array}{c}3 \text {-f hex. } \\
(111)\end{array}$ & recons. \\
\hline $\mathrm{Pt} / \mathrm{Pt}(111)[21]$ & $\begin{array}{c}>0, \text { close } \\
\text { to recons. }\end{array}$ & $\begin{array}{c}3 \text {-f hex. } \\
(111)\end{array}$ & $\begin{array}{c}\text { poss. } \\
\text { recons. }\end{array}$ \\
\hline
\end{tabular}

Table 1: Compilation of experimental results on equilibrium shapes of epitaxial systems with threefold or sixfold symmetry for which a reasonable comparison with the results of our simulations can be performed, as discussed in the text. Abbreviations used: Pref.: Preferred; Comm: Comments; 3-f: threefold; 6-f: sixfold; hex.: hexagonal; triang.: triangular; recons: reconstructed; poss.: possibly; kinet: kinetics. The systems $\mathrm{Co} / \mathrm{Pt}(111), \mathrm{Au} / \mathrm{Au}(111)$ show a positive surface strain due to surface reconstructions in which additional rows of atoms are inserted. The $\mathrm{Pt}(111)$ surface is also prone to a similar surface reconstruction at the growth temperatures required for achieving equilibrium shapes. See text and cited References for details.

Co monolayer and allows the island to expose only (111)faceted steps in the equilibrium shape [30, in agreement with our prediction.

In further different metallic epitaxial systems, triangular islands have been found, but these most probably do not correspond to thermodynamic equilibrium shapes and are determined instead by kinetics (e.g. different diffusion coefficients along different step types), although both facts might be related. Examples are $\mathrm{Co} / \mathrm{Ru}(0001) 31$ and $\mathrm{Co} / \mathrm{Cu}(111)$ [32. For the tensile Co islands on $\mathrm{Ru}(0001)$, a careful structural determination by combined low-energy electron diffraction and microscopy has shown that the preferred microfacets are of the (100) type 33, in agreement with our result. Threefold hexagonal islands of $\mathrm{Au}$ on $\mathrm{O} / \mathrm{Ru}(111)$ have also been observed upon annealing, evolving from dentritic shapes [34].

In homoepitaxial islands, the tensile stress present in unreconstructed hexagonal surfaces 35] should result in a hardly significant predominance of (100) steps according to our model. However, in these conditions, some more subtle effects such as those arising from the degree of $d$ band filling [36] can be significant. Nearly regularhexagonal islands have been observed for $\mathrm{Cu}(111)$ and $\mathrm{Ag}(111)$ 37. However, in the reconstructed $\mathrm{Au}(111)$ surface or in $\mathrm{Pt}(111)$, reconstructed at the temperatures and fluxes necessary to achieve growth of equilibrium shapes [38, the stress should be compressive and the predicted threefold hexagonal islands with preferred (111)-

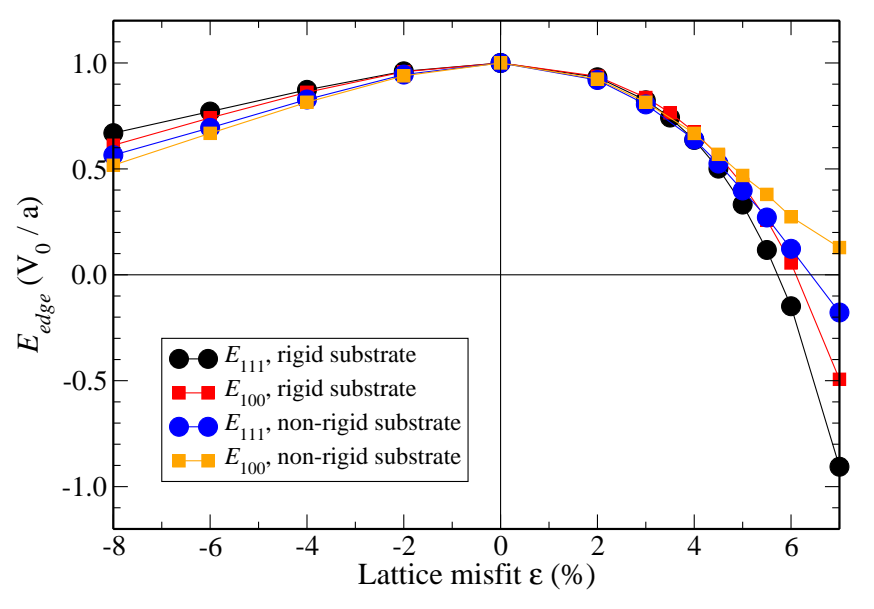

Fig. 5: Dependence of the energies of $A$ and $B$ steps, in units of $V_{0} / a$, on the lattice misfit, calculated by allowing atoms in the topmost atomic layer of the substrate to relax in the same way as the atoms in the island, as explained in the text. In addition, the data for the rigid substrate shown in Fig. 2 are included for comparison.

faceted steps have indeed been observed [21].

Considering more complex systems beyond the applicability of our simple model, triangular equilibrium shapes have been also observed for graphene islands on $\mathrm{Ni}(111) 39$ and $\mathrm{WS}_{2}$ on $\mathrm{Au}(111)$ 40. Epitaxial graphene domains evolve towards triangular equilibrium shapes with only one of the two possible zig-zag edge orientations, while for $\mathrm{WS}_{2}$, triangles exposing either the so-called Sand W-edges have been observed. Epitaxial strain in both systems is very small. The reason for an energetic preference of one step type is not evident and interdiffusion of $\mathrm{Ni}$ and $\mathrm{S}$ adsorption on the edges of $\mathrm{WS}_{2}$ have been proposed, respectively. An overview of the experimental results discussed above that can be reasonably compared with the results of our simulations is given in Table 1. Although quantitative comparison is difficult in several cases, results are in reasonable agreement with the predictions of our model.

In summary, we have found that the step energies of strained hexagonal monolayer islands depend on the amount of strain induced by the lattice misfit. Edge energies decrease with increasing absolute value of the misfit due to strain relaxation. The effect is much more pronounced in the case of positive misfit owing to the stronger repulsive interatomic forces. The energy of (111)-faceted steps is lower at positive misfits (compressed islands) that of (100)-faceted steps. The ratio is reversed at negative misfits (tensile islands). The equilibrium shape transforms from regular hexagons at zero misfit to islands with threefold symmetric hexagonal shape with increasing misfit. The results can be understood in terms of the different bonding geometries at the two different types of steps and have been found to compare reasonably with available experiments.

This work was supported by projects FIS2008-01431 
and FIS2011-23230 of the Spanish MiCInn.

\section{REFERENCES}

[1] Gibbs J.W., Trans. Connecticut Acad., 3 (1878) 343.

[2] Curie, P., Bull. Soc. Min. France, 8 (1885) 145.

[3] Wulff G., Z. Kristallogr., 34 (() 1901)449.

[4] Herring C., Structure and Properties of Solid Surfaces, edited by Gomer R. and SMith C.S. (University of Chicago Press) 1952, p. 5.

[5] Landau L.D. and Lifshitz E.M., Statistical Physics, Vol. 5 (Addison-Wesley, Reading MA) 1958, p. 460.

[6] Bonzel H.P., Physics Reports, 385 (2003) 1.

[7] Tersoff J. and Tromp R.M., Phys. Rev. Lett., 70 (1993) 2782.

[8] Tersoff J. and Legoues F.K., Phys. Rev. Lett., 72 (1994) 3570.

[9] Müller P. and Kern, R., Surf. Sci., 457 (2000) 229.

[10] Politi P., Grenet G., Marty A., Ponchet A. and Villain J., Phys. Rep., 324 (2000) 271.

[11] Giesen M., Progr. Surf. Sci., 68 (2001) 1.

[12] Burton W. K., Cabrera N. and Frank, F.C., Phil. Trans. Roy. Soc. London, 243 (1951) 299.

[13] Stoyanov S. and Markov I., Surf. Sci., 116 (1982) 313.

[14] Priester C. and Lannoo M., Phys. Rev. Lett., 75 (1995) 93.

[15] Li A., Liu F. and Lagally M.G., Phys. Rev. Lett., 85 (2000) 1922.

[16] Pradhan A., Ma N.-Y. and Liu F., Phys. Rev. B, 70 (2004) 193405.

[17] LiU F., Handbook of Theoretical and Computational Nanotechnology, Vol. 4, edited by Rieth M. and Schommers W. (American Scientific Publishers) 2006, p. 577.

[18] Zandvliet H.T.W. and van Gastel R., Phys. Rev. Lett., 99 (2007) 136103.

[19] Marchenko V.I. and Parshin A.Y, Sov. Phys. JETP, $52(1980) 129$.

[20] Hamilton J.C., Phys. Rev. Lett., 88 (2002) 126101

[21] Michely T. and Comsa G., Surf. Sci., 256 (1993) 217.

[22] Li J, Modelling Simul. Mater. Sci. Eng., 11 (2003) 173.

[23] Markov I., Phys. Rev. B, 48 (1993) 14016.

[24] Markov I. and Milchev A., Surf. Sci., 145 (1984) 313.

[25] Markov I. and Prieto J.E., Kotrla M., PapanicoLAOU N., VVEDENSKY D.D. and Wille L.T. (Editors), Atomistic Aspects of Epitaxial Growth; NATO Science Series II, Vol. 65 (Kluwer, Dordrecht) 2001, p. 411.

[26] Frank F.C. and van der Merwe J.H., Proc. Roy. Soc. London, 198 (1949) 205, 216.

[27] Chernov A.A., Modern Crystallography III; Springer Series in Solid State Sciences, Vol. 36 (Springer, Berlin) 1994.

[28] Röder H., Hahn E., Brune H., Bucher J.-P. and KERN K., Nature, 366 (1993) 141.

[29] Cren T., Rusponi S., Weiss N., Epple M. and Brune H., J. Phys. Chem B, 108 (2004) 14685.

[30] Brune H., Handbook of Surface Science, Vol. 3 (Elsevier, Amsterdam) 2008, sect. 15.

[31] Hwang R.Q., Günther G., Schröder J., Günther S., Kopatzki E. and Behm R.J., J. Vac. Sci. Technol. A, 10 (1992) 1970.

[32] de la Figuera J., Prieto J.E., Ocal C. and Miranda R., Phys. Rev. B, 47 (1993) 13043.
[33] El Gabaly F., Puerta J.M., Klein Ch., SaA A., Schmid A.K., MCCARTy K.F., Cerda J.I. and DE lA Figuera J., New Journal of Physics, 9 (2007) 80.

[34] Schröder J., Günther C., Hwang R.Q. and Behm R.J., Ultramicroscopy, 42 (1992) 475.

[35] H. IвACH, J. Vac. Sci. Technol. A, 12 (1994) 2240.

[36] Papadia S., Desjonquères M.C. and Spanjaard, D., Phys. Rev. B, 53 (1996) 4083.

[37] Giesen M., Steimer Ch. and Ibach H., Surf. Sci., 471 (2001) 80.

[38] Bott M., Hohage M, Michely Th. and Comsa G., Phys. Rev. Lett., 70 (1993) 1489.

[39] Li M., Hannon J.B., Tromp R.M., Sun J., Li J., Shenoy V.B. and Chason E., Phys. Rev. B, 88 (2013) 041402.

[40] Füchtbauer H.G., Tuxen A.K., Moses P.G., Topsoe H., Besenbacher F. and Lauritsen J.V., Phys. Chem. Chem. Phys., 15 (2013) 15971. 